\title{
Characterizing the Impacts of Phasing, Environment, and Temporal Factors on Pedestrian Demand at Traffic Signals
}

\author{
Christopher M. Day, Hiromal Premachandra, and Darcy M. Bullock \\ Purdue University \\ 27 S. Martin Jischke Drive, West Lafayette, IN, 47907
}

\begin{abstract}
There is a need for more and higher quality data on pedestrian demand patterns for a number of applications in planning, transportation engineering, public health, and other areas. It is particularly desirable to better characterize the influence of daily, weekly, and annual variations; the impact of weather and special events; and the effects of changes in pedestrian phasing. This paper proposes and demonstrates a methodology for quantifying the relative demand for pedestrian service at a signalized intersection by using the percent of signal cycles per hour in which the pedestrian phase was actuated. Although this performance measure does not by itself provide a pedestrian count, it can be used as a surrogate to characterize how pedestrian volumes vary due to operating conditions. More importantly, since this technique does not require new sensors, the data can be collected at thousands of intersections across the nation where pedestrian push buttons are in use.
\end{abstract}

This paper documents findings from over a year of data collection at a signalized intersection on a college campus. The effects of daily/weekly/annual variations, special events, weather (temperature and precipitation), seasonal changes in activity patterns, and changes in pedestrian signal phasing are documented. A Tobit model is used to account for the influences of these variables and understand how they co-influence pedestrian activity. The implementation of an exclusive pedestrian phase is associated with a $9 \%$ increase in pedestrian phase utilization at the intersection. This change is associated with a decrease in user cost relative to performing midblock crossings. The modeled impact of snowfall events adds further insight by showing that as the user cost of making midblock crossings increases, pedestrian activity at the intersection increases. 


\section{INTRODUCTION}

This paper presents a simple method for characterizing the relative level of pedestrian demand with regard to seasonal fluctuations, weather, changes in activity patterns, special events, and changes in traffic signal phasing. The proportion of traffic signal cycles in which pedestrian phases are actuated is proposed as a surrogate measure of pedestrian volumes. Although this measure does directly quantify the number pedestrian using the facility, it allows the relative demand between different time periods and operating conditions to be characterized. Most importantly, the methodology can be implemented at thousands of intersections where pedestrian-actuated phases are in operation with minimal cost.

\section{BACKGROUND}

More and better data on pedestrian utilization is needed for planning, safety, and operations applications in transportation engineering, as well as other areas (e.g., public health). At present, methods for developing pedestrian volumes lag behind the corresponding methods for vehicular traffic (1). Models are emerging for predicting demand for pedestrian facilities from surrounding land use (e.g., 2, 3, 4), which may eventually lead to planning analysis tools similar to vehicle trip generation tables. There are few studies on the smaller-scale aspects of pedestrian travel, such as how pedestrian activity varies on a day-to-day basis and with respect to weather, special events, and other factors. While planners currently have access to published seasonal adjustment factors for vehicle traffic, similar numbers are not well characterized for pedestrians at most locations. There is a growing awareness of this disparity as communities continue to become more aware of the importance of non-motorized modes. However, agencies face substantial challenges in obtaining this data.

Perhaps the most substantial challenge to collecting data on pedestrian activity is finding the resources necessary to collect the data. There are emerging technologies for automatic pedestrian counting methods $(5,6,7,8,9,10,11)$ and a desire to develop traffic signal performance measures that characterize pedestrian service $(12,13)$. However, most agencies continue to rely on manual pedestrian counts to characterize pedestrian activity in an area (5). Two recent studies have proposed methodologies that could potentially characterize pedestrian demand effectively over a 
broad region over an extended time period, particularly if the concepts are combined. Scheider et al. (8) proposed a methodology for using automatic pedestrian counts to extrapolate volumes from two-hour manual counts to a weekly basis. Estimated volumes could further be transferred to locations with similar land use characteristics or form the basis of safety analyses. AultmanHall et al. (11) presented the results of automatic pedestrian counts after one year of data collection, permitting the observation of fluctuations with respect to seasonal variations and weather. Combining long-term measurement of variations in pedestrian demand using automatic data collection methods with short-term, high-precision manual counts could form a basis for characterizing pedestrian demand in a large geographic region throughout the year, closing the disparity in planning and engineering resources for vehicle traffic and pedestrian traffic.

This paper proposes an approximate method that could accelerate that process by observing relative changes in pedestrian demand over a long time period using a surrogate measure, the percentage of traffic signal cycles where pedestrian phases are actuated. We envision that this would be combined with short-term manual counts, or by automatic counts (perhaps by equipment that is rotated throughout numerous locations in a jurisdiction) to characterize pedestrian demand for many different conditions.

This methodology has the advantage of being almost immediately deployable at thousands of intersections where pedestrian push buttons are in use. The disadvantages of this methodology are that it does not directly provide pedestrian counts, and it is not applicable to locations where pedestrian phases are not actuated.

A case study is presented where pedestrian phase utilization was tracked between November 2008 through April 2010 at a signalized intersection. Changes in the level of pedestrian activity are characterized with respect to daily, weekly, and annual fluctuations; weather conditions (temperature and precipitation); special events; changes in activity patterns due to the academic calendar of a nearby university; and changes in pedestrian service at the intersection (implementation of an exclusive pedestrian phase). The impact of these factors are reported in detail and further investigated in an econometric model to reveal insights as to how they coinfluence the level of pedestrian activity. 


\section{METHODOLOGY}

\section{Study Location}

The intersection of Northwestern Ave. (US 231) and Stadium Ave. in West Lafayette, Indiana is situated on the northwestern edge of the Purdue University campus. This location experiences many different levels of pedestrian demand, while experiencing moderate to high amounts of vehicular traffic. A map of the intersection is shown in Figure 1. Academic buildings dominate the southwest quadrant of the intersection, while athletic facilities exist to the northwest. There are several businesses on the northeast and southeast quadrant near the intersection, while the rest of the nearby area is residential. Several important pedestrian traffic flow patterns occur at this location:

- Students and university employees cross from residential areas on the east side of the street to the campus on the west side of the street. This includes both residents of those neighborhoods as well as commuters using street parking.

- At the middle of the day (lunch hour), many pedestrians cross Northwestern Avenue to visit restaurants and other businesses on the east side of the street.

- The number of pedestrians during summer break is much less than during the academic year due to drastic changes in activity patterns. Approximately 40,000 students attend Purdue University, the majority of which do not attend classes in summer.

- During special events, large numbers of pedestrians cross Northwestern Avenue to attend football and basketball games after parking in the neighborhood or at the parking garage on Northwestern Avenue. According to a Purdue University Police Department 2009 report, average attendance for the 2008-2009 academic year was 75,000 people including tailgaters for football games, and 12,500 people for basketball games.

The magnitude of the change in activity patterns during summer is characterized by changes in the ridership of a bus route that exclusively serves West Lafayette and passes through the test intersection. The average number of riders for this route for January-April 2009 was 19,075 per month, compared to 8,203 per month for May-July 2009, which is a 57\% reduction [numbers for Route 5 (14)]. 


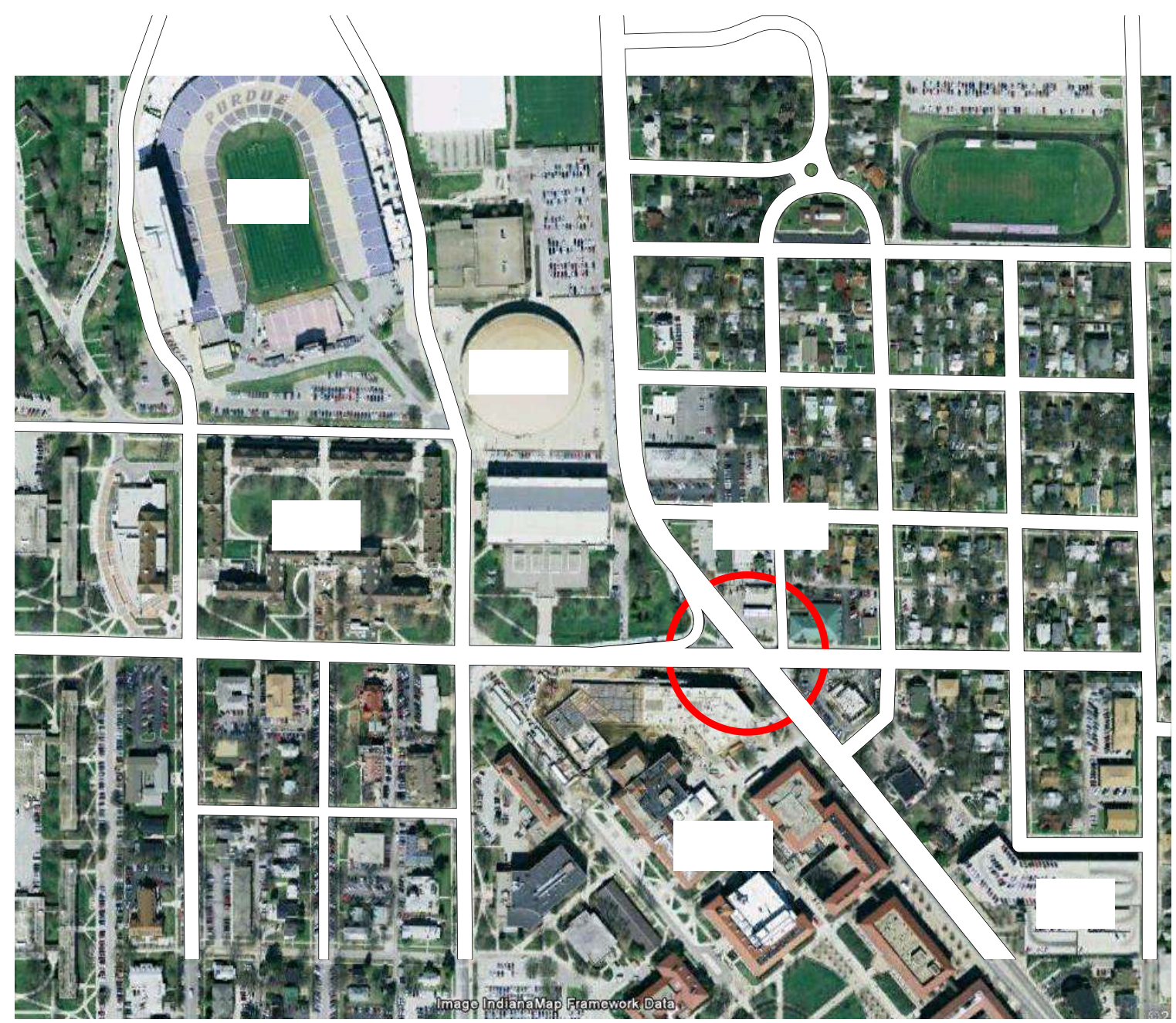

Figure 1. Location of Northwestern and Stadium (West Lafayette, IN) and surrounding features (aerial photo source: Google Earth). 
West Lafayette, Indiana experiences a variety of weather conditions throughout the year. Temperatures range from an average high of $86^{\circ} \mathrm{F}$ in July to an average low of $18.5^{\circ} \mathrm{F}$ in January. About 41 inches of precipitation fall during the year, with about 27 inches of snowfall on average (15).

Figure 2 describes the intersection of Northwestern and Stadium in detail. The layout of lanes and signal phases are shown in Figure 2a. Ring diagrams explaining the signal operation are shown in Figure $2 \mathrm{~b}$ for traditional pedestrian phases and in Figure $2 \mathrm{c}$ for an exclusive pedestrian phase (pedestrian scramble). These diagrams display the sequence of phases during each signal cycle and the phase numbering scheme in use at the intersection.

Pedestrians crossing Northwestern must traverse five lanes of vehicular traffic. The "Ped 4" movement (i.e., the movement adjacent to vehicular phase 4) is a concern at this intersection because eastbound right turning vehicles often fail to yield to pedestrians. The high volume of vehicles that execute the eastbound right turn during green implies headways that are unlikely to allow comfortable pedestrian movements $(12,13)$. In 2009, it was decided to add an exclusive pedestrian phase to this movement to improve conditions. This exclusive pedestrian phase was actuated whenever a pedestrian pushed a button to cross Northwestern (Ped 4 or Ped 8). Pedestrians that pushed only the button to cross Stadium would not receive the exclusive phase, but instead would receive a pedestrian indication that timed concurrent with the adjacent vehicular phase (Ped 2 or Ped 6).

Crossing of Northwestern Avenue is by no means limited to the intersection. It is also very common for pedestrians to cross the street south of the intersection. First, pedestrians negotiate one direction of vehicular traffic, and then stand at the median strip to wait for a safe gap to cross the other direction. It was speculated that the addition of the exclusive pedestrian phase would attract more pedestrians to the intersection to cross Northwestern because of the enhanced convenience of being able to cross both streets and all vehicle movements in one movement. 


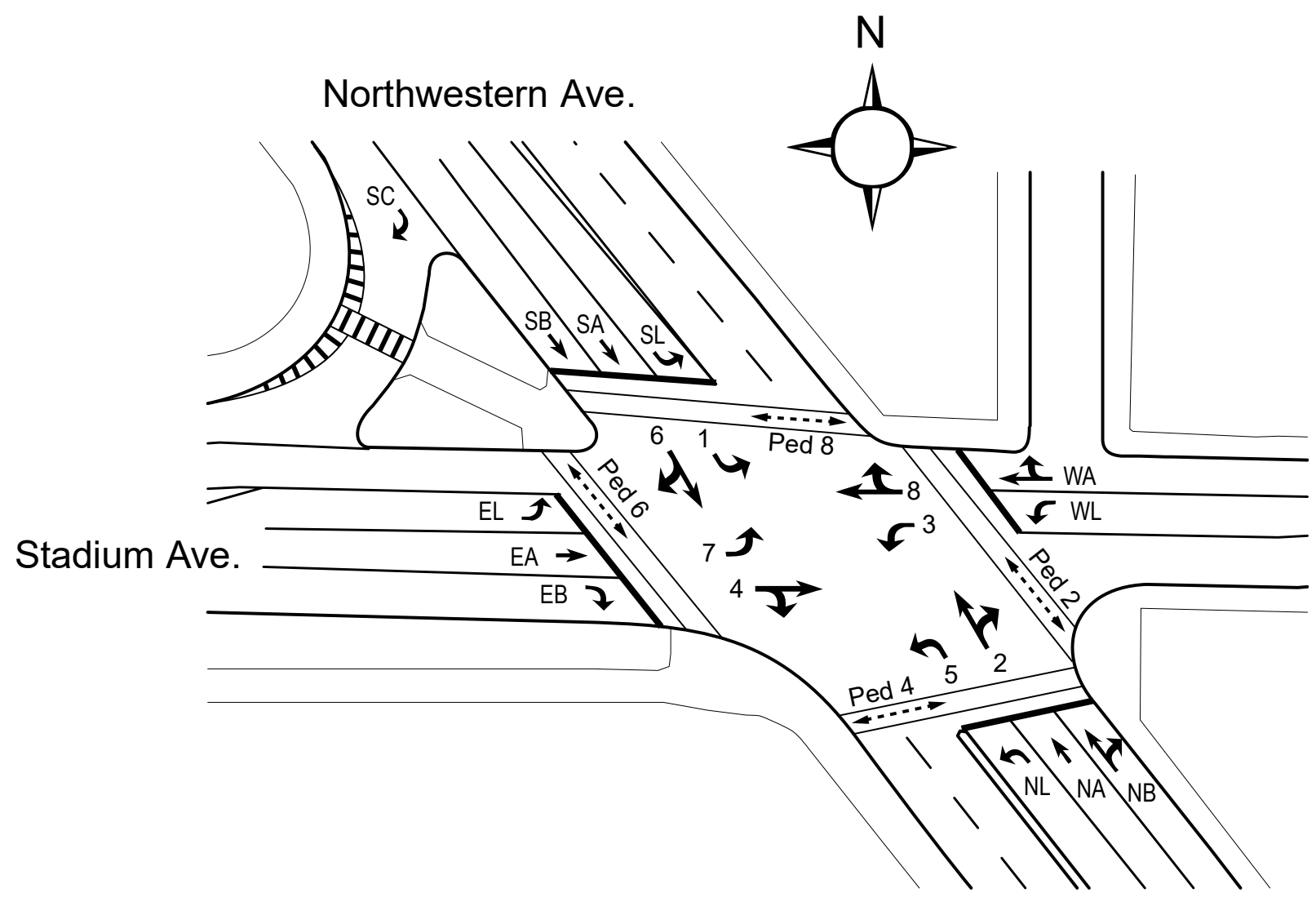

(a) Vehicle and pedestrian phase numbering scheme.

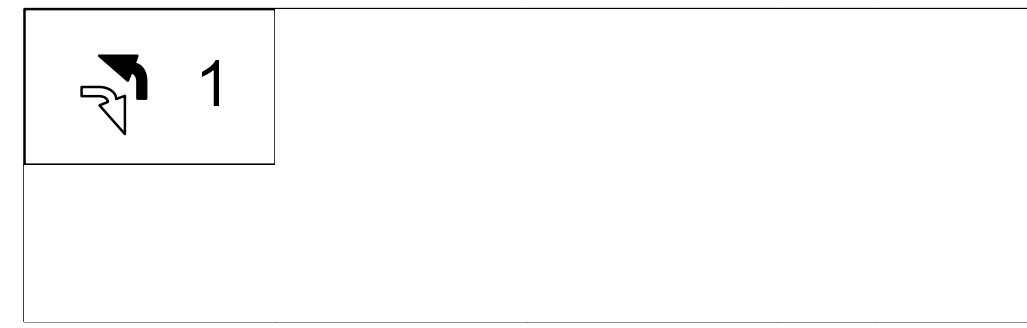

(b) Ring diagram with conventional adjacent pedestrian phases.

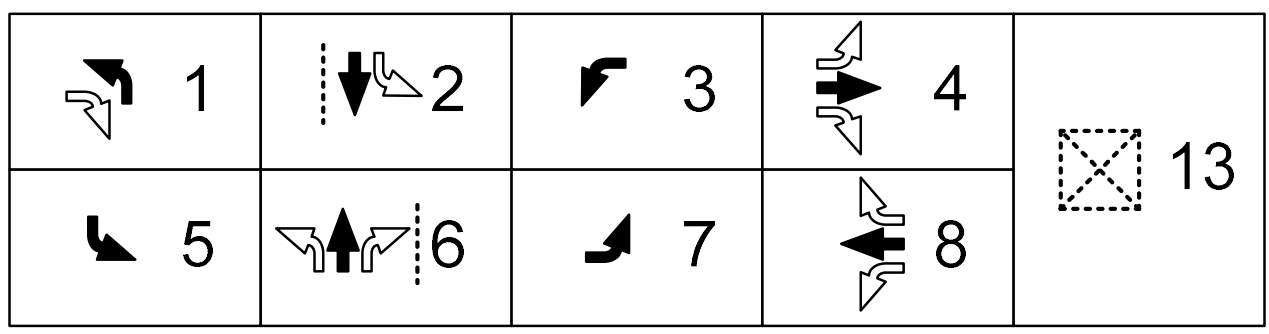

(c) Ring diagram with exclusive pedestrian phase.

Figure 2. Intersection phase configuration at Northwestern and Stadium. 


\section{Data Collection Methodology}

Data was collected at the intersection throughout several intervals spanning November 2008 to April 2010. A log-capable traffic signal controller (16) was used to record the timestamped start and end time of all vehicular and pedestrian phases. Log files were collected via automatic scheduled FTP downloads through a virtual private network (VPN) connection. At this location, a direct fiber optic connection existed between the intersection and the Purdue traffic lab. However, the same signal data logging methodology has been used extensively at numerous remote locations with connectivity established using commercial IP data connections. The cost of implementing similar data collection capability at a typical intersection is summarized in Table 1.

Table 1. Cost of implementation of the data collection methodology.

\begin{tabular}{lr}
\hline Item & Cost \\
\hline One Time Costs & \\
\hline Log-Capable Traffic Signal Controller & \\
(e.g., Econolite ASC/3, Peek ATC) & $\$ 2000$ \\
Wireless Modem & $\$ 500$ \\
VPN Router & $\$ 120$ \\
Installation labor & \\
$\quad(2$ hours at $\$ 100$ per hour) & $\$ 200$ \\
VPN Server & $\$ 200$ \\
Dedicated computer for automatic downloads & $\$ 1000$ \\
Software for executing automatic downloads & $\$ 100$ \\
\hline Total of One-Time Costs & $\$ 4,120$ \\
\hline Annual Costs & $\$ 500 /$ year \\
\hline 12 months of data-only wireless service
\end{tabular}


Cycles were identified from the signal event data from phase 2 end of green. Using these events to divide the timeline into discrete cycles, it was possible to identify cycles where pedestrian phases took place. For this study, we focused on the pedestrian phases that crossed Northwestern Avenue: Ped 4 / Ped 8, or the exclusive pedestrian phase. The reason for doing so was to observe the impact of the exclusive pedestrian phase. The Ped 4 movement is the most challenging for pedestrian and motivation for installing an exclusive pedestrian phase at this location.

In addition to the signal data, weather conditions were obtained from a public database for the nearest geographic location (West Lafayette airport), while the academic calendar and football/basketball game schedule information was obtained from Purdue University's website. All of this data was compiled on an hour-by-hour basis for further analysis. A total of 10,478 data points were obtained. These are summarized in Table 2. 
Table 2. Characteristics of the collected data.

\begin{tabular}{|c|c|c|c|}
\hline Category & Subcategory & Number of Samples & Percentage of Total \\
\hline \multirow{2}{*}{ Pedestrian Phase Type } & Traditional Phases & 1,790 & $17 \%$ \\
\hline & Exclusive Phases & 8,688 & $83 \%$ \\
\hline \multirow{3}{*}{ Weather (Precipitation) } & No Precipitation & 9,431 & $90 \%$ \\
\hline & Rain (Non-frozen precip.) & 753 & $7 \%$ \\
\hline & Snow (Frozen precip.) & 294 & $3 \%$ \\
\hline \multirow{2}{*}{ Time of Day } & $0600-2200$ & 6,984 & $67 \%$ \\
\hline & Other & 3,494 & $33 \%$ \\
\hline \multirow{2}{*}{ Day of Week } & Weekdays & 7,406 & $71 \%$ \\
\hline & Weekends & 3,072 & $29 \%$ \\
\hline \multirow{12}{*}{ Month of Year } & January & 832 & $8 \%$ \\
\hline & February & 672 & $6 \%$ \\
\hline & March & 1,334 & $13 \%$ \\
\hline & April & 816 & $8 \%$ \\
\hline & May & 744 & $7 \%$ \\
\hline & June & 720 & $7 \%$ \\
\hline & July & 744 & $7 \%$ \\
\hline & August & 744 & $7 \%$ \\
\hline & September & 720 & $7 \%$ \\
\hline & October & 744 & $7 \%$ \\
\hline & November & 1,256 & $12 \%$ \\
\hline & December & 1,152 & $11 \%$ \\
\hline \multirow{3}{*}{ Academic Year } & Academic Year & 6,536 & $62 \%$ \\
\hline & Summer Break & 2,568 & $25 \%$ \\
\hline & Other Breaks & 1,374 & $13 \%$ \\
\hline \multirow{3}{*}{ Special Events } & Football game & 160 & $2 \%$ \\
\hline & Basketball game & 156 & $2 \%$ \\
\hline & Other & 24 & $<1 \%$ \\
\hline \multicolumn{2}{|c|}{ Total Number of 1-hour Samples } & 10,478 & $100 \%$ \\
\hline
\end{tabular}




\section{MEASUREMENT RESULTS}

\section{Daily, Weekly, and Annual Variations}

Figure 3 shows the daily level of pedestrian utilization of phases crossing Northwestern. This plot shows the average percentage of cycles per hour (between 6:00 AM and 10:00 PM) for which the pedestrian movements Ped 4 and/or Ped 8 were served, either by traditional adjacent phases (Figure 2b, prior to March 17, 2009) or by exclusive pedestrian phases (Figure 2c, from March 17, 2009). Figure 3a shows all of the observed data, while Figure $3 b$ shows only weekdays where class was in session. Two periods where data collection was temporarily unavailable are indicated by the gray regions in the plots. All data is shown after the implementation of the exclusive pedestrian phase.

The bimodal nature of the data with respect to two variables is clearly shown in Figure $3 \mathrm{a}$. Pedestrian phase utilization is much lower during summer (callout A, approximately mid-May through mid-August) than during the academic year (callout B). Additionally, pedestrian phase utilization is consistently higher during weekdays (callouts A,B) than weekends (C,D), both during summer $(A, C)$ and the academic year $(B, D)$. In Figure 3b, where we eliminate all but days with classes in session, seasonal trends are also visible, as well as the impact of the introduction of the exclusive phase. 


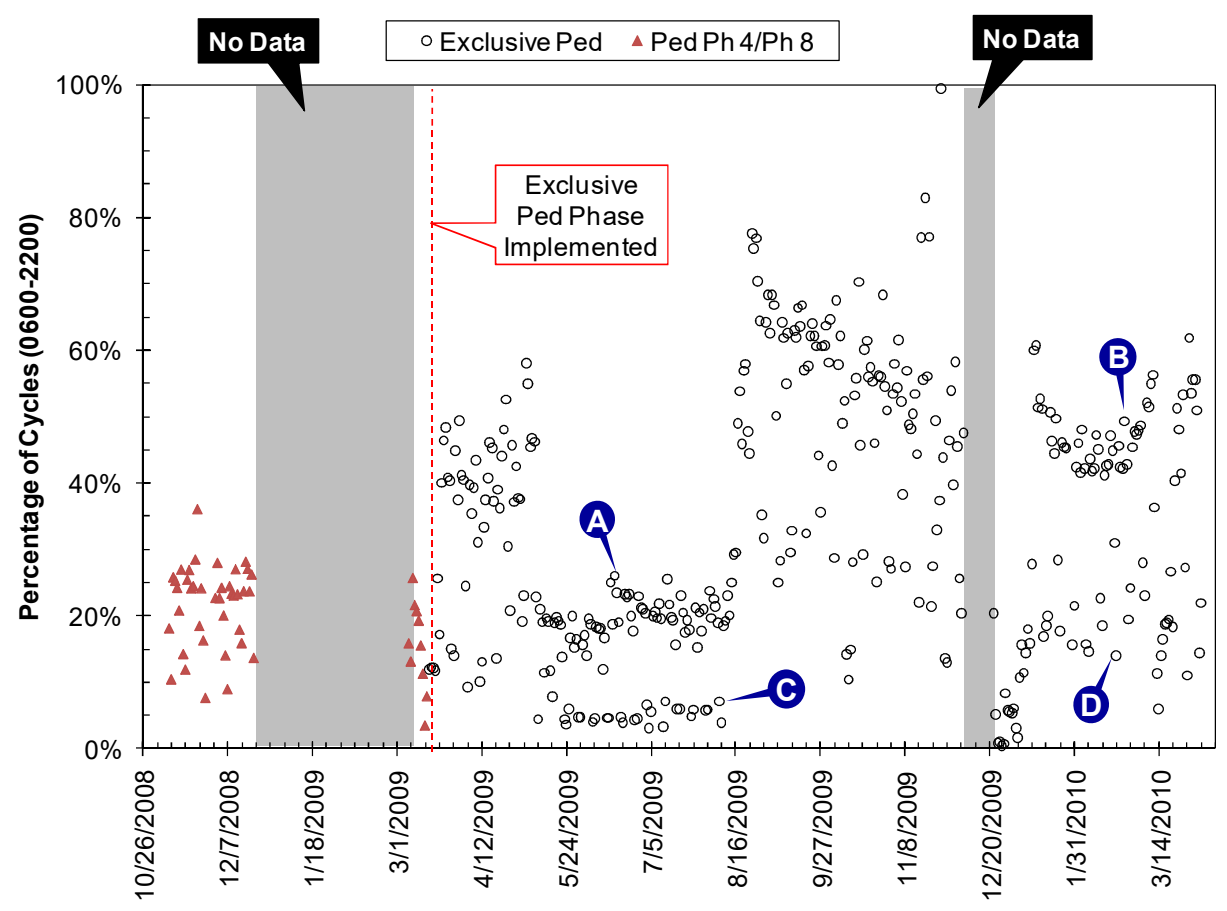

(a) All days when data was collected.

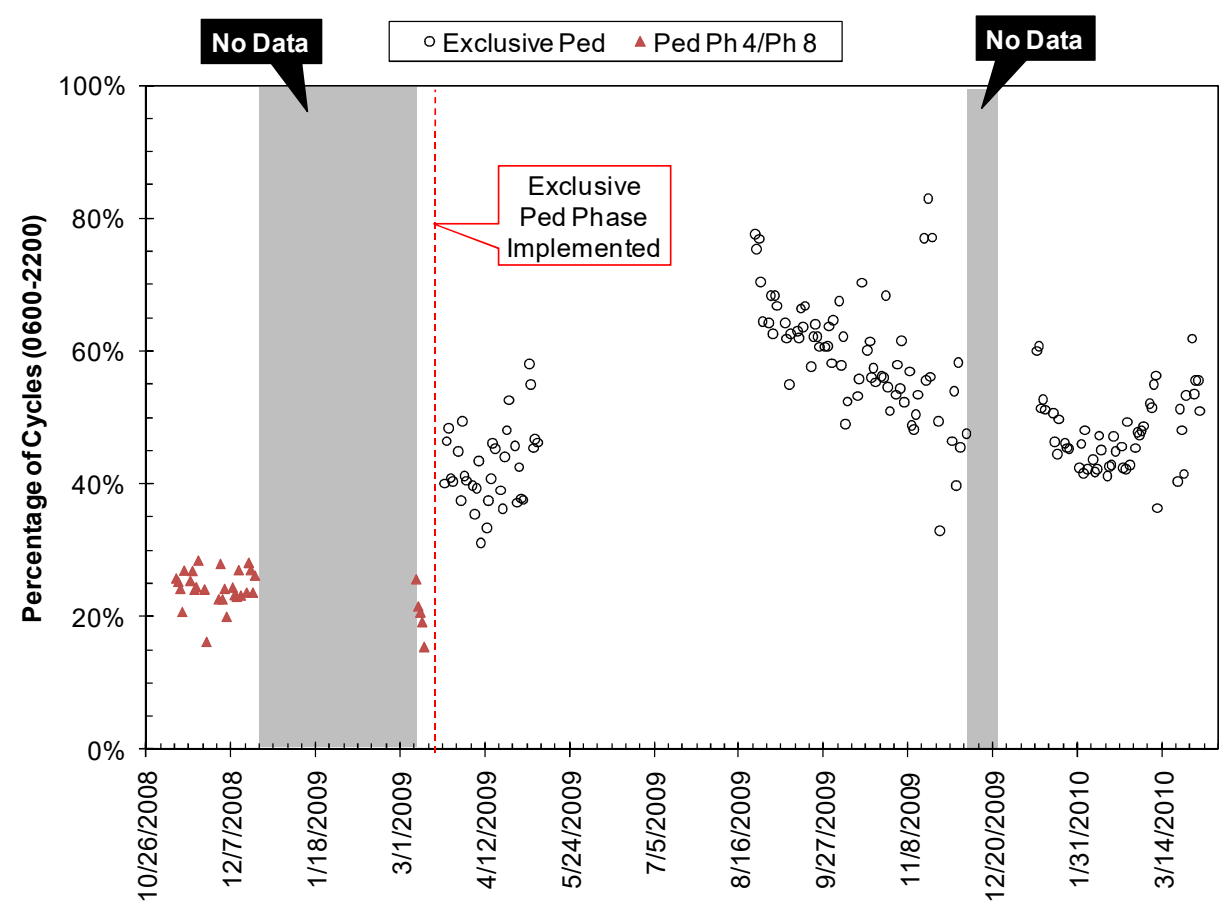

(b) Only days in the academic year with classes in session.

Figure 3. Percentage of cycles with pedestrian phases (during 0600-2200) crossing Northwestern Avenue (West Lafayette, IN). 
Figure 4 examines daily, weekly, and annual variations in greater detail.

- Pedestrian activity is very low in the early morning and late night. Overall trends are similar during both weekdays (Figure 4a) and weekends (Figure 4b). The two series of bars in these plots represent data from the academic year and non-academic year. On weekdays during the summer, ped phase usage is greatest at approximately noon (Figure $4 a)$, while during the academic year the ped phase is sustained from noon through about 6:00 PM. Pedestrian activity is lower on weekends but is still higher during the academic year than otherwise. Interestingly, there is a local spike in pedestrian activity at 1:00am on weekdays (Figure 4b)when classes are in session, the imprint of an activity pattern that clearly does not occur when the student population is absent.

- Figure 4c shows variations in pedestrian activity from 0600-2200, again with two series representing the academic year and non-academic year. The differences between weekdays and weekends, and between the academic year and non-academic year are about the same as that seen in the daily variation. Pedestrian activity is slightly less on Sunday than Saturday; there is very little difference from day to day among weekdays.

- Annual variations in pedestrian activity (during 0600-2200) are shown in Figure 4d. In this plot, the two series represent weekdays and weekends. Changes in pedestrian activity from month to month are largely dominated by student activity, with the least pedestrian activity during June and July and the greatest amount during September.

These results may be compared with yearlong study of pedestrian counts from an automatic pedestrian counter in a downtown area of Montpelier, Vermont (11). Daily variations are similar, with the exception of the late-night activity that occurs in our data during the academic year, most likely because of the activity patterns of university students. The annual variations are considerably different in our data because the activity patterns are so heavily dominated by student activity. This demonstrates the importance of local data collection for characterizing demand. 


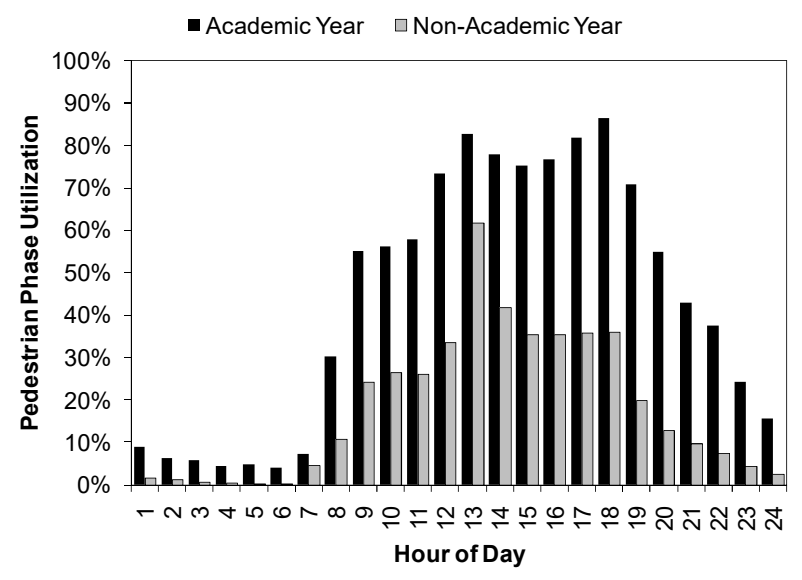

(a) Daily variation (weekdays).

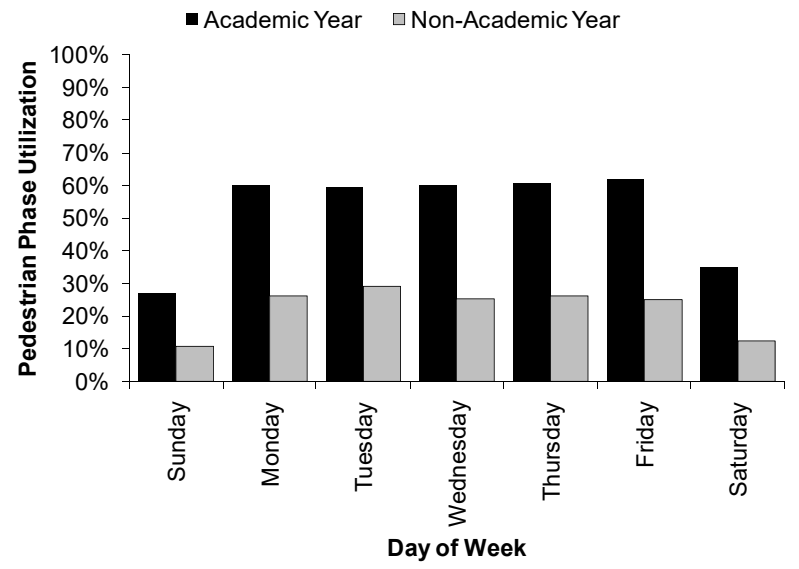

(c) Weekly variation (0600-2200).

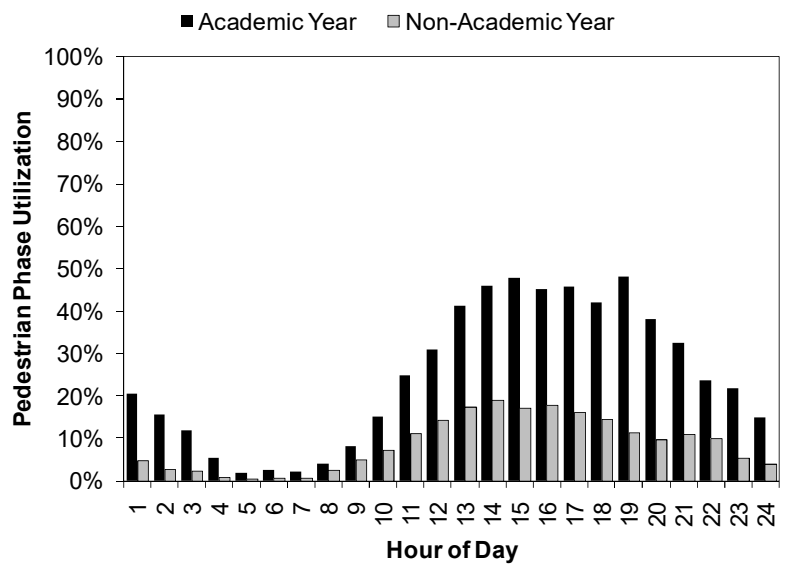

(b) Daily variation (weekends).

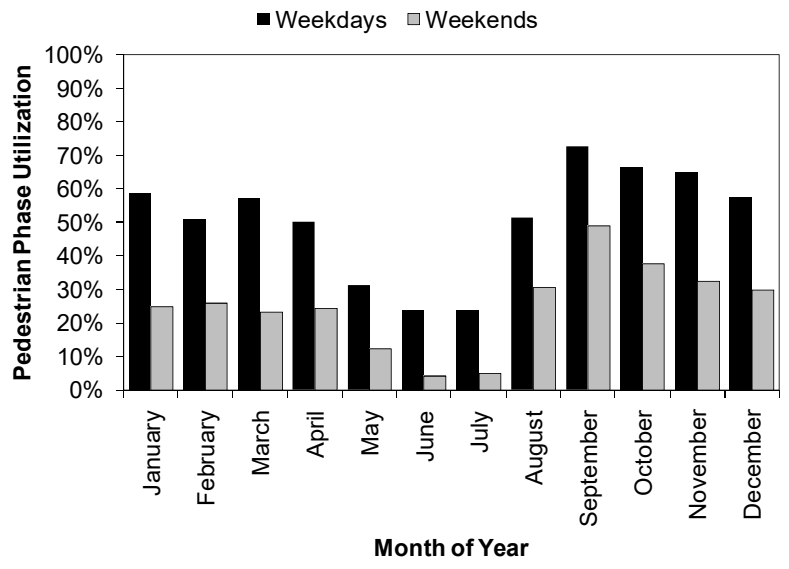

(d) Annual variation (0600-2200).

Figure 4. Daily, weekly, and annual variation in pedestrian phase utilization (all data from after implementation of exclusive pedestrian phase). 


\section{Weather}

Weather conditions were matched for all of the 1-hour samples in the data set. Figure 5 illustrates the relationships between pedestrian phase utilization and temperature (Figure 5a) and precipitation (Figure 5b). Each plot shows two separate series for weekdays and weekends; data are shown for the academic year from 0600-2200, after the implementation of the exclusive pedestrian phase. Pedestrian activity tends to increase with temperature (Figure 5a); there is particularly little pedestrian activity on cold weekends. Pedestrian activity also tends to decreases during snow and rain (Figure $5 \mathrm{~b}$ ). The decreases are stronger on weekends than during weekdays, and the pedestrian phase is activated less often during rain than during snow.

\section{Special Events}

The proximity of the football stadium and basketball arena to the intersection of Stadium and Northwestern offers an excellent opportunity to observe the effects of special events on levels of pedestrian activity. A 1-hour sample was considered to be a "game event" within 8 hours of the beginning of a football game, and within 6 hours of the beginning of a game for a basketball event. For days within the academic year, pedestrian phase utilization averaged $62 \%$ during football game hours compared to $47 \%$ for non-football game hours (excluding the early morning hours). The corresponding numbers for basketball games are $51 \%$ and $48 \%$. The impact of football games is evidently more substantial than that of basketball games, which is not unexpected given the relative attendance numbers discussed earlier.

\section{Changes in Signal Operation}

Figure 6 compares the percentage of cycles with pedestrian phases with conventional ped phases (Figure 2b) in use and with an exclusive ped phase (Figure 2c) in use. The data in this graph are from hours between 0600-2200 in November 2008 (conventional) and November 2009 (exclusive), and exclude special events, school breaks, and inclement weather. By November 2009, the exclusive phase had been in operation for eight months. The change in the percentage of cycles with pedestrian phases is shown at the top of the graph. For weekdays, average utilization increased from $45 \%$ to $63 \%$ while for weekends it increased from $17 \%$ to $30 \%$. This 
data clearly shows that the addition of the exclusive pedestrian phase led to a moderate increase in the number of pedestrians using the intersection to cross Northwestern.

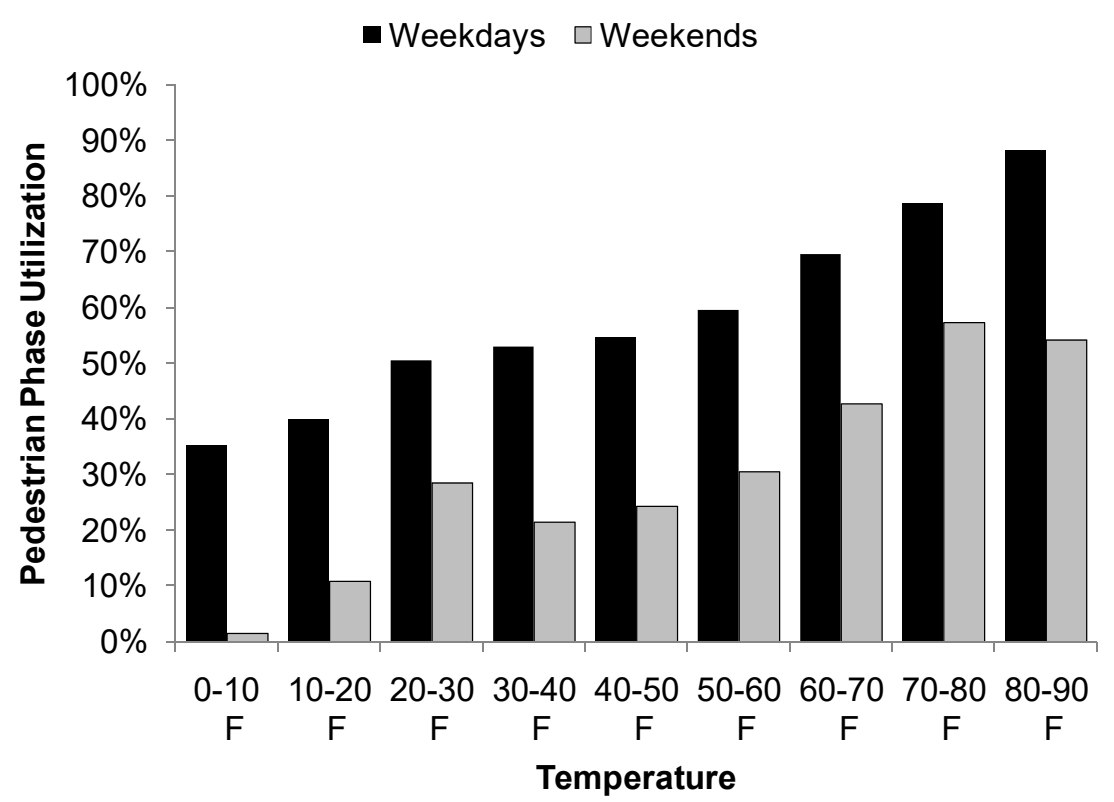

(a) Temperature.

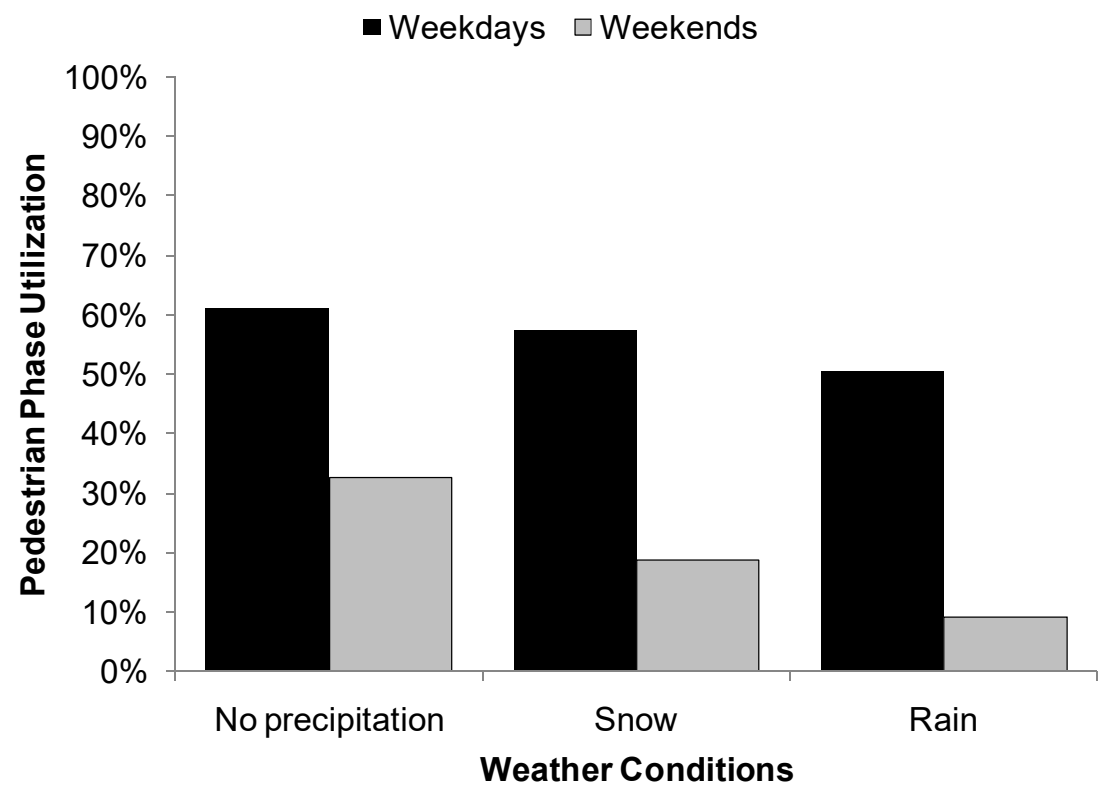

(b) Precipitation.

Figure 5. Variation in pedestrian phase utilization due to weather effects (all data from 0600-2200 during academic year after implementation of exclusive pedestrian phase). 


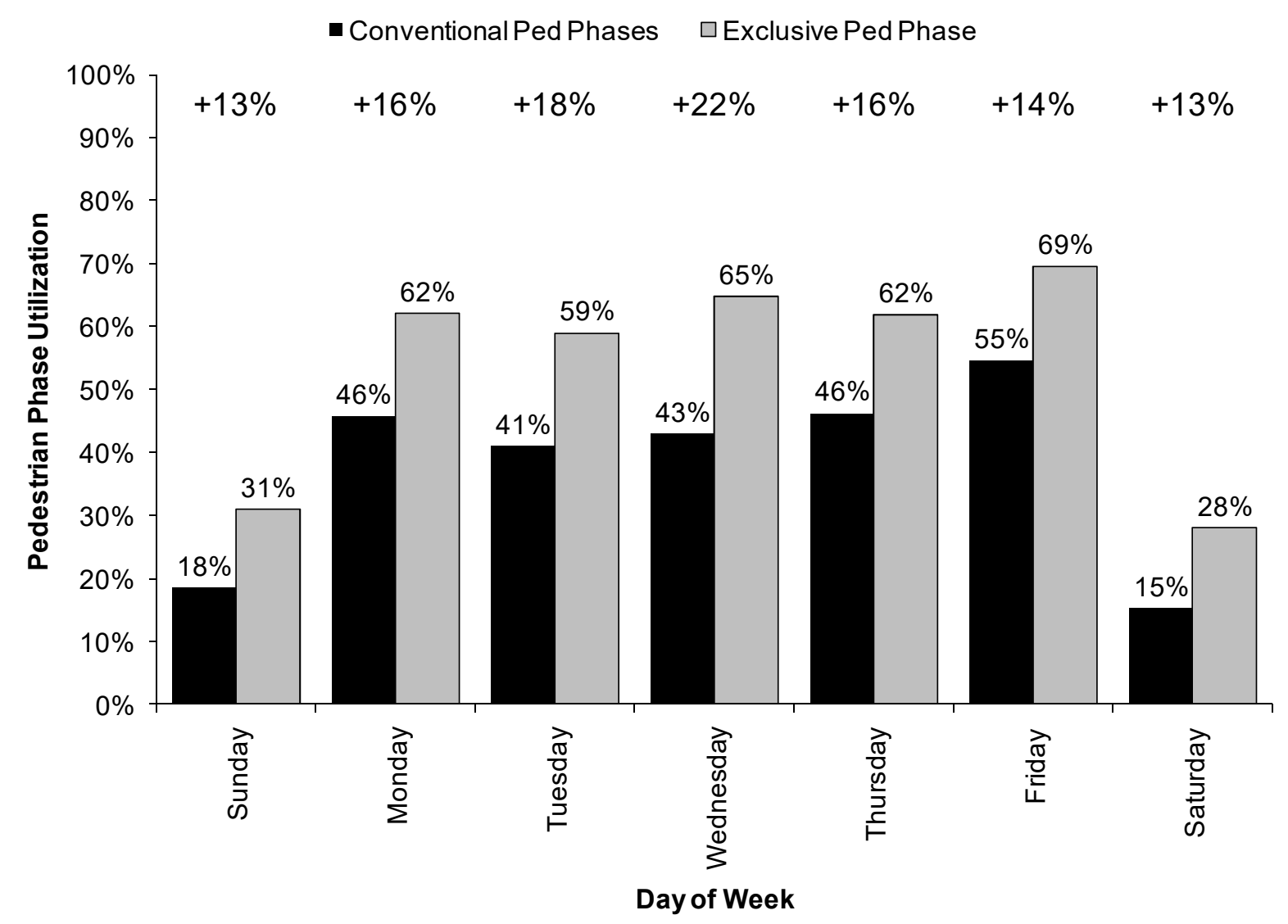

Figure 6. Percentage of cycles with pedestrian phases crossing Northwestern Avenue before and after exclusive pedestrian phase implementation (Data from November 2008 and November 2009, 0600-2200, excluding special events, breaks, and inclement weather). 


\section{ECONOMETRIC ANALYSIS}

\section{Model Development}

To make a better sense of how several different variables concurrently influence pedestrian utilization, we estimate a statistical model and develop coefficients for the variables encoding the effects. The dependent variable, the percentage of cycles with pedestrian phases, is interval censored because it can only have values between $0 \%$ and $100 \%$. There are consequentially many data points clustered at $0 \%$ and $100 \%$. An ordinary least squares regression would not be appropriate for this dependent variable because it would be heavily skewed by the clustered data points at the ends of the possible range. An alternative model is a Tobit regression (17), which is intended for a continuous, censored dependent variable. Despite there being many modeled variables with censored data, Tobit regression has seen relatively little use in transportation, mainly in safety $(18,19,20,21)$ and economics $(22,23)$.

The data from the 10,478 1-hour observations were encoded into a series of dependent variables listed in Table 3. The majority of the variables were encoded as indicator variables (i.e., "1" means that the condition was true, while " 0 " means the condition was not true), with the exception of temperature, which was simply included as degrees Fahrenheit. Using temperature as a continuous variable resulted in better model fit than numerous indicator variables based upon temperature. Table 4 shows the model characteristics indicating the effects of the variables, their significance, and overall model characteristics. The goodness of fit is given by the Maddala pseudo- $R^{2}(24)$

$$
\begin{array}{lr}
\text { Maddala pseudo- } R^{2}=1-\exp [-L R T / N] & \text { Equation 1 } \\
L R T=2[L L(\beta)-L L(0)] & \text { Equation 2 }
\end{array}
$$

where $L R T$ is the likelihood ratio test statistic, $L L(\beta)$ is the log likelihood of the model at convergence, $L L(0)$ is the log-likelihood if the coefficients are constrained to zero, and $N$ is the number of observations. The pseudo- $R^{2}$ value of 0.550 is not an unreasonable value for a large number of samples and data that is behavioral in nature. 
Table 3. Descriptive statistics of variables used in the Tobit model.

\begin{tabular}{|c|c|c|c|c|}
\hline Independent Variable & Min & Max & Mean & St. Dev. \\
\hline $\begin{array}{l}\text { Indicator of pedestrian phase configuration } \\
\text { 1: exclusive ped phase; } 0 \text { : conventional ped phases }\end{array}$ & 0 & 1 & 0.842 & 0.365 \\
\hline $\begin{array}{l}\text { Indicator of weekend } \\
\text { 1: Saturday/Sunday; 0: other day of week }\end{array}$ & 0 & 1 & 0.293 & 0.455 \\
\hline $\begin{array}{l}\text { Indicator of break (weekday during academic calendar } \\
\text { without classes in session) } \\
\quad \text { 1: break; } 0 \text { : no break }\end{array}$ & 0 & 1 & 0.131 & 0.338 \\
\hline $\begin{array}{l}\text { Indicator of summer break } \\
\text { 1: summer break; } 0 \text { : not summer break }\end{array}$ & 0 & 1 & 0.245 & 0.430 \\
\hline $\begin{array}{l}\text { Indicator of August } \\
\text { 1: August; 0: other month }\end{array}$ & 0 & 1 & 0.071 & 0.257 \\
\hline $\begin{array}{l}\text { Indicator of "daytime" hours (6:00 AM - 10:00 PM) } \\
\text { 1: daytime hours; 0: other times }\end{array}$ & 0 & 1 & 0.667 & 0.471 \\
\hline $\begin{array}{c}\text { Indicator of "lunchtime" hours }(11: 00 \mathrm{AM}-1: 00 \mathrm{PM}) \\
\text { 1: lunchtime hours; 0: other times }\end{array}$ & 0 & 1 & 0.083 & 0.277 \\
\hline $\begin{array}{c}\text { Indicator of "PM peak" hours (3:00 PM - 6:00 PM) } \\
\text { 1: PM Peak hours; 0: other times }\end{array}$ & 0 & 1 & 0.125 & 0.331 \\
\hline $\begin{array}{l}\text { Indicator of weekend nights }(10: 00 \mathrm{PM}-2: 00 \mathrm{AM}) \\
1 \text { : weekend nights; } 0 \text { : other times }\end{array}$ & 0 & 1 & 0.049 & 0.216 \\
\hline Temperature (Degrees Fahrenheit) & -0.9 & 93.0 & 49.637 & 19.221 \\
\hline Indicator of Rain (1: rain; 0: no rain) & 0 & 1 & 0.072 & 0.258 \\
\hline Indicator of Snow (1: snow; 0: no snow) & 0 & 1 & 0.028 & 0.165 \\
\hline Indicator of football game (1: game; 0 : no game) & 0 & 1 & 0.015 & 0.123 \\
\hline Indicator of basketball game (1: game; 0: no game) & 0 & 1 & 0.015 & 0.121 \\
\hline
\end{tabular}


Table 4. Estimation of a Tobit model to describe pedestrian phase utilization.

\begin{tabular}{|c|c|c|c|}
\hline Independent Variable & Coefficient & $t$-value & $P$-value \\
\hline Constant term & -0.177 & -18.094 & $<0.001$ \\
\hline $\begin{array}{l}\text { Indicator of pedestrian phase configuration } \\
\text { 1: exclusive ped phase; } 0 \text { : conventional ped phases }\end{array}$ & 0.0938 & 13.182 & $<0.001$ \\
\hline $\begin{array}{l}\text { Indicator of weekend } \\
\text { 1: Saturday/Sunday; 0: other day of week }\end{array}$ & -0.236 & -41.252 & $<0.001$ \\
\hline $\begin{array}{l}\text { Indicator of break (weekday during academic calendar without } \\
\text { classes in session) } \\
\qquad \text { 1: break; 0: no break }\end{array}$ & -0.269 & -33.845 & $<0.001$ \\
\hline $\begin{array}{l}\text { Indicator of summer break } \\
\text { 1: summer break; 0: not summer break }\end{array}$ & -0.381 & -53.024 & $<0.001$ \\
\hline $\begin{array}{l}\text { Indicator of August } \\
\qquad 1: \text { August; } 0 \text { : other month }\end{array}$ & 0.147 & 15.281 & $<0.001$ \\
\hline $\begin{array}{l}\text { Indicator of "daytime" hours (6:00 AM - 10:00 PM) } \\
\text { 1: daytime hours; 0: other times }\end{array}$ & 0.431 & 69.470 & $<0.001$ \\
\hline $\begin{array}{c}\text { Indicator of "lunchtime" hours (11:00 AM - 1:00 PM) } \\
\text { 1: lunchtime hours; 0: other times }\end{array}$ & 0.191 & 22.938 & $<0.001$ \\
\hline $\begin{array}{c}\text { Indicator of "PM peak" hours (3:00 PM - 6:00 PM) } \\
\text { 1: lunchtime hours; 0: other times }\end{array}$ & 0.179 & 25.029 & $<0.001$ \\
\hline $\begin{array}{c}\text { Indicator of weekend nights (10:00 PM - 2:00 AM) } \\
\text { 1: lunchtime hours; } 0 \text { : other times }\end{array}$ & 0.305 & 23.667 & $<0.001$ \\
\hline Temperature (Degrees Fahrenheit) & 0.00333 & 19.028 & $<0.001$ \\
\hline Indicator of Rain (1: rain; 0: no rain) & -0.0563 & -6.240 & $<0.001$ \\
\hline Indicator of Snow (1: snow; 0: no snow) & 0.0394 & 2.694 & 0.007 \\
\hline Indicator of football game (1: game; 0 : no game) & 0.272 & 14.659 & $<0.001$ \\
\hline Indicator of basketball game (1: game; 0: no game) & 0.147 & 7.913 & $<0.001$ \\
\hline Number of observations & & 10,478 & \\
\hline Log likelihood at Convergence & & 1726.62 & \\
\hline Log likelihood at Zero & & -2461.60 & \\
\hline Maddala Pseudo- $R^{2}$ & & 0.550 & \\
\hline
\end{tabular}




\section{Analysis}

The indicator variable shown in Table 4 for the exclusive pedestrian phase had a positive coefficient, which agrees with the increase in pedestrian activity related to the exclusive phase that was observed earlier in the raw data. The coefficient of the variable corresponding to the exclusive pedestrian phase, 0.0938 , indicates that pedestrian phase usage increases by $9.38 \%$ after the addition of the feature. The magnitude of the coefficient is greater than variables based on weather effects. The number is slightly less than what is observed in the raw data (Figure 6) because of the influences of other variables in the model.

Three calendar-related variables were included to indicate weekends, breaks during the academic year, summer break, and the month of August. The weekend and break variables were highly significant with large negative coefficients, reflecting the strong decrease in pedestrian activity during weekends and breaks compared to other time periods, as discussed earlier and illustrated by Figure 4. The August variable captures the effects of students returning to campus, where pedestrian activity increases both during the portion of the month during summer break, and the portion after the start of classes.

Four time-of-day variables were defined indicating whether the percentage of cycles is obtained during the "daytime" (defined as 6:00 AM to 10:00 PM), "lunchtime" (11:00 AM to 1:00 PM). a "PM peak" time period (3:00 PM to 6:00 PM), or weekend nights (10:00 PM to 2:00 AM on weekends only). Each of these variables is highly significant, and has a fairly large positive coefficient, indicating the higher levels of pedestrian activity during these times of day. The daytime variable is the most significant variable among all the variables in this model, reflecting the fundamental difference in activity between day and night common to all times of the year (Figure 4a, Figure 4b). The lunchtime and PM peak variables capture local peaks during their respective time periods where pedestrian phase utilization is higher still. The weekend night indicator captures the local spike in pedestrian activity that takes place in the early morning hours, particularly during the academic year (Figure 4c). 
Temperature was included as a continuous variable in the model. The coefficient of 0.00333 indicates an increase in the amount of pedestrian utilization by $0.3 \%$ of cycles per hour for each one-degree Fahrenheit increase in temperature. Pedestrian activity is greater during warmer weather. The model results are consistent with observations from the raw data (Figure 5a).

Two indicator variables for weather conditions were included: one for rain and one for snow. Both of these variables were significant, although less so than the others in the model. The coefficient for rain was negative, indicating that there is less utilization of pedestrian phases when rain occurred. This is in agreement with Figure 5b. The coefficient for snow, however, is positive, indicating that snow the effect of increasing pedestrian utilization. There are, therefore, some conditions under which utilization of pedestrian phases at the intersection increases because of snow. Perhaps this can be explained by the accumulation of snow along the shoulders and median strip on Northwestern Avenue, making it difficult for pedestrians to cross the street by stopping on the median, and encouraging them to use the marked crosswalk at the intersection.

Three indicator variables for special events were also included in the model. These reflected football games, basketball games, and when the first two weeks of classes took place. These three conditions reflected times of heightened pedestrian activity, as indicated by the large positive coefficients and high significance. The magnitude of these effects are about as strong as the day-to-day peaking at lunchtime and during the PM peak. Football games are a stronger generator of pedestrian activity than basketball games, agreeing with the prior discussion of the raw data and the relative attendance of the events. 


\section{Impact of Change in Pedestrian Service}

The increase in pedestrian activity in response to the change in intersection operations can be viewed as an economic interaction of supply and demand (25), as illustrated by Figure 7. The improvement in pedestrian service is equivalent to the movement of the price-volume curve from "System A" to "System B" in the curve, with the area DEBA corresponding to the user benefit (reduction in user cost) and $A F G B$ corresponding the amount of latent demand uncovered by the change in service. The exclusive pedestrian phase reduces user cost by providing a portion of the signal cycle with reduced pedestrian-vehicle conflicts as well as by allowing pedestrians to cross diagonally.

While it is clear that the exclusive pedestrian phase induced more pedestrian usage of the intersection, it seems unlikely that the demand for pedestrian trips across Northwestern Avenue increased as a result of the pedestrian phase. A more realistic explanation is that the increase in pedestrian utilization likely corresponds to a decrease in midblock pedestrian crossings elsewhere. While the exclusive pedestrian phase decreases user costs at the intersection, the characteristics of midblock crossings and their associated user costs have remained unchanged. As a consequence, more of the demand for crossing Northwestern was met by the intersection.

Similar effect can be observed in the impact of snow conditions. In the Tobit regression, the overall effect of the snow indicator variable was to increase pedestrian activity; this is illustrative of the interaction between intersection crossings and the midblock crossings. Snow makes midblock crossings more difficult by reducing the number of places on the median where crossing is feasible (i.e., pedestrians are disinclined to stand at snow or ice covered patches of the median in close proximity to two moving traffic streams). This may be interpreted as an increase in the cost of making a midblock crossing. As this cost increases, pedestrian activity at the intersection increases because it takes on more of the demand to cross the street. This observation agrees with findings by Muraleethan et al., who reported changes in pedestrian routing due to winter weather $(26)$. 


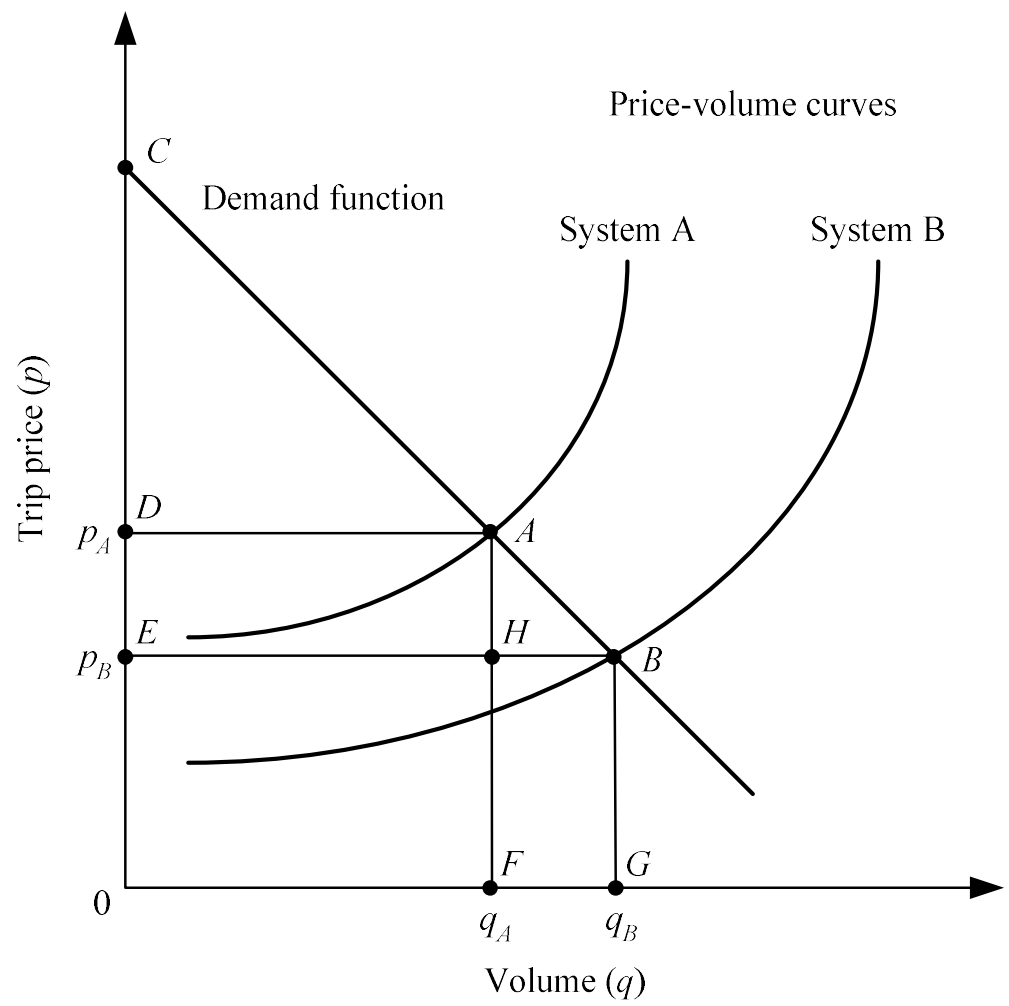

Figure 7. Economic concepts associated with changes in service in a transportation system, after Wohl and Hendrickson (25).

\section{CONCLUSIONS}

This paper proposed and demonstrated a methodology for quantifying the relative amount of pedestrian activity at a signalized intersection by tabulating the percentage of signal cycles per hour in which a pedestrian phase was actuated. While this performance measure does not directly use pedestrian counts, it can be used to characterize the relative change in demand, and offers the advantage of being implementable without installing additional infrastructure. The usefulness of the performance measure was demonstrated by documentation of changes in pedestrian utilization relative to daily, weekly, and annual variations; weather conditions (temperature and precipitation); special events; seasonal changes in activity patterns; and changes to pedestrian service at the intersection (implementation of an exclusive pedestrian phase). A Tobit model was estimated on this data to exemplify how these factors co-influenced the level of pedestrian utilization. The improvement of pedestrian service by implementation of the exclusive phase was associated with a $9.4 \%$ increase in utilization of the phase. This is interpreted economically as a 
decrease in user cost at the intersection relative to unchanging user cost for midblock crossings. This interpretation is consistent with the model findings with respect to snow conditions. Snow conditions lead to an increase in pedestrian activity at the intersection because of an increased cost of making midblock crossings due to the accumulation of snow in the path of those crossings.

\section{ACKNOWLEDGMENTS}

This work was supported by the Joint Transportation Research Program administered by the Indiana Department of Transportation and Purdue University. The contents of this paper reflect the views of the authors, who are responsible for the facts and the accuracy of the data presented herein, and do not necessarily reflect the official views or policies of the sponsoring organizations. These contents do not constitute a standard, specification, or regulation. 


\section{REFERENCES}

1. Porter, C., J. Suhrbier, and W.L. Schwartz. "Forecasting Bicycle and Pedestrian Travel: State of the Practice and Research Needs." In Transportation Research Record No. 1674, Transportation Research Board of the National Academies, Washington, D.C., 1999, pp. 94-101.

2. Ewing, R. and R. Cervero. "Travel and the Built Environment: A Synthesis.” In Transportation Research Record No. 1780, pp. 87-113, Transportation Research Board of the National Academies, Washington, D.C., 2001.

3. Moudon, A.V., P.M. Hess, J.M. Matlick, and N. Pergakes. "Pedestrian Location Identification Tools: Identifying Suburban Areas with Potentially High Latent Demand for Pedestrian Travel." In

Transportation Research Record No. 1818, pp. 94-101, Transportation Research Board of the National Academies, Washington, D.C., 2002.

4. Pulugurtha, S.S. and S.R. Repaka. "Assessment of Models to Measure Pedestrian Activity at Signalized Intersections." In Transportation Research Record No. 2073, pp. 39-48, Transportation Research Board of the National Academies, Washington, D.C., 2008.

5. Schneider, R., R. Patton, J. Toole, and C. Raborn. Pedestrian and Bicycle Data Collection in United States Communities: Quantifying Use, Surveying Users, and Documenting Facility Extent. Pedestrian and Bicycle Information Center, Highway Safety Research Center, University of North Carolina, Chapel Hill, January 2005.

6. Green-Roesel, R., M.C. Diogenes, D.R. Ragland, and L.A. Lindau. Effectiveness of a Commercially Available automated Pedestrian Counting Device in Urban Environments: Comparison with Manual Counts. Traffic Safety Center, University of California, Berkeley, 2007.

7. Bell, A. Technology Innovations: Infrared Bicyclist and Pedestrian Counter. In Bike/Ped Professional: Journal of the Association of Pedestrian and Bicycle Professionals, pp. 4-5, 2006.

8. Schneider, R.J., L.S. Arnold, and D.R. Ragland. A Methodology for Counting Pedestrians at Intersections: Using Automated Counters to Extrapolate Weekly Volumes from Short Manual Counts. Traffic Safety Center, University of California, Berkeley, 2008.

9. Ling, B., S. Tiwari, Z. Li, and D.R.P. Gibson. "A Multi-Pedestrian Detection and Counting System Using Fusion of Stereo Camera and Laser Scanner." Proc., SPIE Optics and Photonics, California, August 2010.

10. Schneider, R.J., L.S. Arnold, and D.R. Ragland. "Pilot Model for Estimating Pedestrian Intersection Crossing Volumes." In Transportation Research Record No. 2140, Transportation Research Board of the National Academies, Washington, D.C., 2009, pp. 13-26.

11. Aultman-Hall, L., D. Lane, and R.R. Lambert. "Assessing Impact of Weather and Season on Pedestrian Traffic Volumes.” In Transportation Research Record No. 2140, pp. 35-43, Transportation Research Board of the National Academies, Washington, D.C., 2009.

12. Hubbard, S.M.L., D.M. Bullock, and C.M. Day. "Integration of real-time pedestrian performance measures into existing infrastructure of traffic signal system." Transportation Research Record No. 2080, Transportation Research Board of the National Academies, Washington, DC, pp. 37-47, 2008.

13. Hubbard, S.M.L., D. M. Bullock, and F.L. Mannering, "Right-Turns on Green and Pedestrian Level of Service: A Statistical Assessment," ASCE Journal of Transportation Engineering, Vol. 135, No. 4, pp. 153-159, April 2009.

14. City Bus of Greater Lafayette, Indiana ridership reports. http://www.gocitybus.com/ridership.html. Accessed on July 15, 2010. 
15. National Weather Service climate data. http://www.weather.gov/climate. Accessed on July 15, 2010.

16. Smaglik, E.J., Sharma, A., Bullock, D.M., Sturdevant, J.R., and Duncan, G. "Event-Based Data Collection for Generating Actuated Controller Performance Measures." Transportation Research Record No. 2035, Transportation Research Board of the National Academies, Washington, DC, pp. 97-106, 2007.

17. Tobin, J. "Estimation of relationships for limited dependent variables." Econometrica, Vol. 26, pp. 24-36, 1958.

18. Weiss, A.A. "The effects of helmet use on the severity of head injuries in motorcycle accidents." J. Am. Stat. Assoc., Vol. 87, pp. 48-56, 1992.

19. Talley, W.K. "Safety Investments and Operating Conditions: Determinants of Accident PassengerVessel Damage Cost.” Soc. Methods. Res. Vol. 31, pp. 364-388, 1995.

20. Nolan, A. "The Determinants of Urban Households' Transport Decision: a Microeconomic Study Using Irish Data." Presentation to the $7^{\text {th }}$ Spring Meeting of Young Economists, Paris, France, 2002.

21. Anastasopoulos, P.C., A.P. Tarko, and F.L. Mannering. "Tobit Analysis of Vehicle Accident Rates on Interstate Highways." Accident Analysis and Prevention, Vol. 40, pp. 768-775, 2008.

22. Scheraga, C.A. "Operational Efficiency Versus Financial Mobility in the Global Airline Industry: a Data Envelopment and Tobit Analysis." Transportation Research Part A, Vol. 38, pp. 383-404, 2004.

23. Garcia Sanchez, I.M. "Technical and Scale Efficiency in Spanish Urban Transport: Estimating with Data Envelopment Analysis.” Advances in Operations Research, Article ID 721279, 2009.

24. Maddala, G.S., Limited-dependent and Qualitative Variables in Econometrics. Cambridge University Press, New York, 1983.

25. Wohl, M. and C. Hendrickson. "Transportation Investment and Pricing Principles." John Wiley and Sons, 1985.

26. Muraleetharan, T., K. Meguro, T. Adachi, T. Hagiwara, and S. Kagaya. "Influence of Winter Road Conditions and Signal Delay on Pedestrian Route Choice in Japan's Snowiest Metropolis." In

Transportation Research Record No. 1939, pp. 145-153, Transportation Research Board of the National Academies, Washington, D.C., 2005. 